\title{
Economia do Futebol: Uma Abordagem Através do Modelo Agente-Principal
}

Marcelo de C. Griebeler ${ }^{1}$

Diego Baldusco²

Resumo: O presente trabalho modela teoricamente o problema da divergência de objetivos existente no futebol brasileiro como uma relação de agente-principal. $\mathrm{O}$ principal, a torcida, delega para o agente, o dirigente, a tarefa da produção de vitórias e resultados positivos. Contudo, nem sempre o dirigente do clube deseja maximizar o número de vitórias. Entre seus objetivos podem estar manter o orçamento do clube equilibrado ou obter mais sócios, por exemplo. Os principais resultados do trabalho encontram evidência empírica: podemos entender as agressões a dirigentes, jogadores e técnicos de futebol e as invasões de campo protagonizadas por torcedores.

Palavras chaves: Economia do Futebol; Agente-Principal; Informação Assimétrica

1 Professor adjunto da Universidade Federal do Rio Grande do Sul - UFRGS, Faculdade de Ciências Econômicas, Departamento Economia e Relações Internacionais, Avenida João Pessoa 52, Centro Histórico, Porto Alegre - RS, Brasil. CEP: 90040-00o - Fone: 55 (51) 3308-3324. E-mail: marcelo.griebeler@ufrgs.br. 2 Mestre em Quantitative Economics na Universität Konstanz. E-mail: diegobaldusco@gmail.com. 
Abstract: This paper models theoretically the problem of divergence of goals in the Brazilian soccer as a relationship of principal-agent. The principal, the fans, delegates to the agent, the manager, the task of producing wins and results. However, not always the manager of the club wants to maximize the number of victories. Among their goals may be to keep the budget balanced or to obtain more club members, for example. The main paper's results meet empirical evidence: we can understand the attacks on managers, players and soccer coaches and invasions of the field played by fans.

Keywords: Soccer Economics; Principal-Agent; Asymmetric Information

JEL: D82, D86

\section{Introdução}

A Economia do Esporte é um ramo relativamente novo do conhecimento. Somente na década de 50 surgiram os primeiros trabalhos tratando do assunto. A preocupação principal dos precursores desta nova área recaía basicamente sobre dois aspectos do esporte, notadamente a cláusula de reserva do beisebol americano e a discussão sobre qual é o agente econômico relevante no esporte, se o próprio time ou a liga em que este faz parte . $^{3}$.

Embora existam algumas tentativas de usar modelos econômicos aplicados ao futebol, a maior parte dos trabalhos teóricos na literatura mundial é voltada para o beisebol. De fato, modelos com fundamentos microeconômicos são preteridos a formulações empíricas, com a grande maioria do trabalho sendo focados em estimações de demanda por jogos (nos estádios e na televisão), por exemplo. Essa não é apenas uma tendência na literatura internacional, pois apesar do Brasil ser reconhecido mundialmente como "o país do futebol", muito pouco tem se estudado sobre a face econômica dessa atividade em âmbito nacional.

Essa lacuna por si só já seria uma motivação bastante forte para um trabalho que a preenchesse, contudo, as peculiaridades do futebol brasileiro fornecem uma razão adicional para modelarmos a relação econômica entre os agentes envolvidos no meio futebolístico. Considere os recentes atritos entre torcedores e jogadores, treinadores e dirigentes de seus clubes. Esse não é um fato comum em outros países, onde manifestações contra as direções dos clubes, quando ocorrem, são pacíficas e respeitosas. Ao que parece, no Brasil, existe uma divergência entre o que a direção ${ }^{4}$ dos clubes objetiva e o que os torcedores desejam. 
Algumas notícias publicadas na imprensa nacional nos últimos anos revelam a gravidade dessa falta de sincronia entre torcida e dirigentes. Veja, por exemplo, "Invasão no Fazendão desestabiliza elenco do Bahia", publicada no site do UOL Esportes no dia 21 de agosto de 2008. Nesse episódio, as manifestações chegaram às vias de fato, segundo o UOL Esportes, “(...) depois de sofrerem agressões de membros da organizada Terror Tricolor durante o treinamento, jogadores e comissão técnica do Bahia voltaram às atividades nesta quinta-feira". Outro time de massa, o Flamengo, também sofreu com a revolta dos seus torcedores, dessa vez até explosivos foram utilizados. Conforme a manchete do dia 5 de agosto de 2008 no site do Globoesporte, "Torcedores invadem treino, protestam e atiram bomba nos jogadores do Fla".

As ofensas não se restringem a jogadores, dirigentes e técnicos também são alvos. Ricardo Rotenberg, dirigente do Botafogo, por exemplo, foi agredido dentro da sede do clube. A notícia foi publicada no site do Globoesporte no dia 13 de maio de 2008 sobre o título "Agressão a dirigente deixa Bebeto de Freitas irritado". Vanderlei Luxemburgo, então técnico do Palmeiras, também sofreu tentativa de agressão de torcedores. Como cita a matéria do site do Estadão do dia 14 de novembro de 2008: "O técnico Vanderlei Luxemburgo entrou em confronto com membros da Mancha Alviverde na noite desta sexta-feira (...)”. A notícia intitulada "Torcida tenta agredir Vanderlei Luxemburgo em aeroporto" é mais um exemplo da má relação entre torcida e direção dos clubes.

Portanto, existe uma divergência de objetivos no futebol brasileiro que torna conflituosa a relação entre os torcedores e os dirigentes de seus clubes. $\mathrm{O}$ presente trabalho modela teoricamente esse problema como uma relação de agente-principal. $O$ principal, a torcida, delega para o agente, o dirigente, a tarefa da produção de vitórias e resultados positivos. Contudo, nem sempre o dirigente do clube deseja maximizar o número de vitórias. Ele pode querer manter o orçamento do clube equilibrado, para isso vendendo os melhores atletas do elenco. Outra possibilidade é seu objetivo ser maximizar o número de sócios e, assim, se engajar em campanhas publicitárias. De fato, existem diversos outros objetivos que o dirigente pode perseguir que não a maximização de resultados positivos.

O trabalho, dessa forma, tem dois objetivos. O principal deles é modelar a relação torcida-direção através da teoria da agência. Sob um ambiente de informação assimétrica, como é o caso no futebol brasileiro, onde existe pouca transparência nas ações dos dirigentes e mesmo sobre sua habilidade de gestor esportivo, este problema se torna mais delicado. Nesse caso, a torcida não sabe o tipo do dirigente, se eficiente ou ineficiente, e deve escolher como dar os incentivos corretos para que eles produzam bons resultados. Já o objetivo secundário é apresentar uma breve retrospectiva e revisão, além de introduzir a literatura da Economia do Esporte em âmbito nacional, a fim de incentivar mais pesquisas nessa área. O Brasil, sem dúvida, é um campo extraordinário de experimentos no tema, em especial no que tange ao futebol. 
O trabalho é dividido como segue. Além desta introdução, a seção 2 apresenta uma revisão da literatura sobre Economia do Esporte. Atenção especial é dada aos trabalhos teóricos, sem deixar, entretanto, de mencionar os estudos seminais na área. O modelo teórico é desenvolvido e comentado na terceira seção. Primeiramente são discutidas suas implicações sob informação simétrica. Esse resultado é usado posteriormente como benchmarking. A seguir, a principal modelagem do trabalho é apresentada e discutida, qual seja, sob informação assimétrica. Os principais resultados teóricos do trabalho encerram a seção. Por fim, a conclusão resume as principais colaborações do artigo e apontam direções para trabalhos futuros.

\section{Revisão da literatura}

\subsection{Origens da Economia do Esporte}

O primeiro passo na tentativa de analisar a Economia do Esporte foi dado por Rottenberg (1956). Seu estudo trata do mercado de trabalho no beisebol americano e sua principal preocupação é avaliar as conseqüências da cláusula de reserva. Especificamente, se esta contribui positivamente para equidade de forças entre os times, como era argumentado na época, ou não. A cláusula de reserva, em termos gerais, dava ao atleta a oportunidade de escolher o clube com o qual assinaria o contrato de trabalho somente uma vez. A partir daí, seu contrato pode ser renovado ou então ele pode ser negociado com outro clube. Ambas as decisões são exclusivas do time. O principal defesa da cláusula de reserva na época dizia que esta impediria que as equipes mais ricas adquirissem os melhores jogadores.

Para atingir tal objetivo, Rottenberg (1956) utiliza o ferramental microeconômico, mas não sem notar importantes características do setor e fazer importantes hipóteses. Entre as peculiaridades do meio esportivo, o autor destaca a natureza de monopsônio no mercado de trabalho, a importância da incerteza sobre o resultado para a presença de público nos estádios e a natureza do produto e da demanda. Com relação às hipóteses, a principal delas, que mais a frente dará origem à divisão entre o segmento europeu e americano na Economia do Esporte, é a de enxergar o time como maximizador de lucro.

O seu principal resultado é conhecido na literatura como o Princípio da Invariância. Este estabelece que, assumindo-se como verdadeira a lei dos retornos marginais decrescentes, então todo time contratará jogadores até igualar seu retorno marginal ao seu custo marginal. Como resultado, ter-se-ia a igualdade de forças nas ligas. Em outras palavras, é um ataque à cláusula de reserva e uma defesa do livre mercado. Essa conclusão também é condicional a hipótese da importância da incerteza do resultado. Segundo Rottenberg (1956), 
a principal fonte de receitas dos times é a proveniente dos ingressos e sua venda será maior quanto maior for a incerteza sobre o resultado da partida. Dessa forma, é racional para a equipe não possuir um time muito mais forte que os adversários, pois isso diminuiria suas receitas.

O artigo que dá sequência ao tema da Economia do Esporte é o de Neale (1964). Sua atenção é voltada para a discussão de qual é a "firma" relevante no esporte, se o time em si ou a liga na qual este está incorporado. Contrariando Rottenberg (1956), o autor indica que a liga é o agente econômico que se deve levar em conta no esporte. Seu argumento baseia-se na produção conjunta do produto, as partidas, pelos times, de forma que eles devem cooperar para obter êxito. Portanto, cada time individualmente não pode ser visto como uma firma, e sim o seu conjunto, a liga. Junte-se a isso o fato de a liga comandar as regras de competição, mobilidade dos jogadores e entrada e saída de times.

Respondida esta primeira pergunta, Neale (1964) questiona o porquê da ausência de competição entre ligas do mesmo esporte, dado que elas são as firmas relevantes no sentido econômico. Sua resposta indica que as peculiaridades de custo e demanda criam monopólios naturais, ou seja, é ineficiente para uma liga individualmente ofertar o mercado inteiro. Pelo lado dos custos, as principais características, nesse sentido, são os custos médios de longo prazo quase horizontais e a potencial quebra do poder de monopsônio dos times pela entrada de ligas concorrentes. Já no lado da demanda, o autor destaca especificamente o beisebol: além da demanda direta por ingressos existe a indireta, para todos aqueles que acompanham o campeonato. Esta última seria maximizada quando se tem monopólio.

Como conclusão do trabalho é indicada a necessidade de se considerar as peculiaridades supracitadas do esporte (ligas e times) por legisladores, cortes de justiça e poder público, quando da escolha coletiva para políticas específicas para o setor e, ainda, da decisão de qual a regulamentação antitruste adotar.

O terceiro estudo clássico sobre o tema é o de Sloane (1971), pioneiro ao tratar do esporte europeu e britânico. $\mathrm{O}$ artigo se engaja na discussão de qual a unidade de decisão relevante no esporte, questionando o argumento de Neale (1964). O autor cita o caso do futebol inglês, onde órgãos do governo estabelecem parâmetros dentro dos quais os clubes podem operar livre e independentemente. Ainda sobre esta questão, se o produto total (número de partidas disputadas) é regulado, isto meramente reflete o interesse comum dos clubes. Além disso, o autor dá o exemplo de cartéis. Estes tomam decisões de preços e quantidades conjuntas, mas nem por isso passam as ser uma única firma.

Entretanto, a principal contribuição do artigo é modelar o time como um maximizador de utilidade e não mais de lucro, como fez Rottenberg (1956). O autor alega que no caso do futebol inglês lucros são raros, ao contrário do que se vê nos esportes americanos. Os motivos por trás dos tomadores de decisão do clube podem ser status, poder, prestígio ou mesmo entusiasmo pelo esporte. 
Dessa forma, a função objetivo do time a ser maximizada é uma utilidade, restrita, possivelmente, a uma equação de solvência financeira. Outras razões apontadas para a utilização da hipótese da maximização de utilidade foram: o grande desejo de proprietários, diretores, jogadores e torcida é o sucesso em termos de vitórias; quanto maior o número de vitórias maior a receita de bilheteria (contrariando Rottenberg, 1956); o lucro ainda sim pode entrar como argumento na função de utilidade; muitos clubes podem desejar apenas "sobreviver", vendendo jogadores e; a "saúde" ou qualidade da liga entra na função de utilidade representando a interdependência entre os clubes.

Apesar da desvantagem de usar uma modelagem que se ajusta a quaisquer objetivos, sem uma base teórica consistente, o trabalho dá significantes insights e contrapõe os seus predecessores nas suas conclusões. Por exemplo, o argumento de que os retornos decrescentes deveriam evitar a acumulação de bons atletas nos clubes mais ricos (defesa em favor do livre mercado) pode não se manter sob maximização de utilidade. De fato, a idéia de que incentivos voltados para o lucro ajudam a manter a equidade de forças entre clubes pobres e ricos não é válida. Por fim, é indicado que regulação garantindo livre mercado pode ser mais forte se clubes não forem maximizadores de lucro ${ }^{5}$.

\subsection{Principais trabalhos teóricos}

Uma interessante abordagem à economia do esporte é feita por El-Hodiri e Quirk (1971). A fim de investigar em que extensão as exceções à regulamentação antitruste norte-americana abertas ao esporte são justificáveis, os autores estruturam um modelo econômico teórico. Uma de suas principais motivações para o artigo é baseada nas constantes e históricas disputas judiciais envolvendo ligas, times e órgãos de imprensa (televisão e rádio). Segundo o autor, devido às similaridades existentes, as conclusões do trabalho são aplicáveis ao soccer, ao beisebol, ao basquete e os hockey.

O modelo desenvolvido toma como objetivo a maximização do valor presente descontado do fluxo de lucro líquido por parte dos times e incorpora certas peculiaridades do mercado esportivo. Essas peculiaridades, na época, eram restrições legais, tais como a cláusula de reserva, e aparecem no problema como restrições. Como hipótese adicional, é associada a cada jogador e, consequentemente, a cada time uma quantidade de skills, que depreciam ao longo do tempo. O time obtém receita através do comércio de jogadores e, principalmente, através da venda de ingressos para jogos (em casa e fora).

5 O trabalho de Sloane (1971) inaugurou uma divisão na Economia do Esporte, notadamente entre América do Norte e Europa. Seu artigo parece apontar muitas diferenças entre os mercados dos esportes americanos, tais como beisebol e basquete, por exemplo, e o futebol europeu. As principais diferenças frequentemente citadas são entre os torcedores, entre as estruturas das ligas e entre os objetivos dos times. Essa divisão acabou por dar origem a muitos trabalhos, tanto de um lado como de outro do Atlântico. Para uma discussão sobre o assunto ver Fort (2000). 
Após resolver o problema do time utilizando controle ótimo, El-Hodiri e Quirk (1971) chegam à conclusão de que, sob as restrições legais impostas, a equalização de força entre os times não é compatível com a maximização de lucro. Essa compatibilidade só aconteceria em casos especiais, os quais são claramente violados empiricamente nos quatro esportes estudados. Por fim, é sugerida uma estrutura descentralizada que poderia garantir equalização de forças.

Um dos raros trabalhos a utilizar a modelagem agente-principal em Economia do Esporte é o de Miceli (2004). Sua preocupação é em modelar o mercado de trabalho da liga de beisebol profissional norte-americana. Em especial, sua atenção também é voltada para a cláusula de reserva, a qual impõe que os jogadores formados por uma equipe qualquer permaneçam no seu clube de origem por um período determinado de tempo antes de se transferir. $\mathrm{O}$ beisebol possui um mercado de jogadores muito peculiar, no sentido de que os atletas necessitam de um grande treinamento nos seus clubes de origem para se tornarem hábeis. É raro jogadores chegarem "prontos" aos grandes times.

A maior parte da literatura dá ênfase ao papel distributivo da cláusula, qual seja, de evitar que grandes equipes, com maior poder financeiro, concentrem os melhores jogadores. Seguindo na direção oposta, Miceli (2004) procura em seu trabalho uma racionalidade econômica para a cláusula de reserva. Definindo o atleta profissional de beisebol como principal e seu time como agente, ele demonstra que existe um trade-off para o jogador: pesar os benefícios de um potencial salário alto em outra equipe (grande mobilidade, ausência de cláusula de reserva) e as vantagens de um maior período de treinamento no time de origem e uma melhora individual (com cláusula de reserva).

O modelo estabelece que o jogador não sabe quanto é gasto em treinamento pelo seu time. Esta é a relação de agência entre as partes. Apesar dessa incerteza, o atleta pode ter incentivo a permanecer no time de origem recebendo um salário inferior ao que receberia em um time maior devido aos benefícios futuros do seu treinamento, notadamente uma maior habilidade e uma contratação por uma boa equipe. Os resultados do trabalho corroboram esta possibilidade e, adicionalmente, sugerem que a lucratividade dos times não é necessariamente sinal de colusão entre os proprietários, como sugeria a literatura, e sim um resultado da vontade dos jogadores.

Giovanetti et al. (2006) também apresenta uma abordagem teórica para Economia do Esporte, embora a sua ênfase recaia na parte empírica. Neste estudo, um modelo microeconômico de decisão individual é utilizado para suportar um exercício econométrico sobre a fidelidade das torcidas dos clubes participantes do Campeonato Brasileiro de Futebol, no ano de 2004. A ideia dos autores é investigar se a decisão dos torcedores de ir ao estádio assistir a partida do seu time é influenciada pela probabilidade de vitória do mesmo. Em caso de resposta afirmativa, considera-se o torcedor como infiel. 
Dando mais ênfase a modelagem teórica, temos que os torcedores são definidos como maximizadores de utilidade e suas preferências são definidas em relação à quantidade de dinheiro em mãos e na escolha de ir ao estádio assistir a partida. $\mathrm{O}$ ambiente quase-linear é utilizado para explicitar a pequena influência do preço do ingresso no orçamento individual. Além disso, existem três estados da natureza possíveis para o torcedor, vitória, derrota e empate. O problema passa então a ser maximizar a sua utilidade esperada, com as probabilidades dos três estados da natureza podendo variar de torcedor para torcedor e mesmo para um mesmo indivíduo. As restrições são a tradicional orçamentária e de que o somatório das probabilidades seja igual a um.

Como o modelo teórico é apenas um meio que suporta as estimações econométricas, as conclusões a que chegam Giovanetti et at. (2006) são todas empíricas. Apenas a título de curiosidade, os times que apresentaram as torcidas mais infiéis no período estudado foram Atlético-MG, Grêmio e Internacional.

\section{Modelo 6}

Considere a torcida de um clube de futebol (o principal) que quer delegar aos dirigentes do clube (o agente) a produção de resultados positivos ${ }^{78}$. Por ser não observável, assumiremos que a variável resultados positivos pode ser medida por uma Proxy $m$, que representa a quantidade monetária obtida pelo clube com premiações e títulos. Perceba que quanto mais resultados positivos o clube alcança, mais recebe em prêmios, patrocínios e rendas de televisão. É importante destacar que não estamos incluindo aqui nenhum tipo de transferência do torcedor para o clube, somente fontes externas, como as já citadas. A utilidade para o principal de $\mathrm{m}$ unidades é $\mathrm{V}(\mathrm{m})$, onde $\mathrm{V}^{\prime}>\mathrm{O}, \mathrm{V}^{\prime \prime}<\mathrm{o}$ e $\mathrm{V}(\mathrm{o})=0$. A utilidade marginal dos bons resultados do clube é, assim, positiva e estritamente decrescente para a torcida.

Faremos a hipótese simplificadora de que existem apenas dois tipos de dirigentes, os eficientes e os ineficientes ${ }^{9}$. Eles diferem no seu custo de produção variável $\theta$, o qual é sua informação privada, ou seja, o torcedor não observa

6 Esta seção está baseada em Laffont e Martimort (2002).

7 Seremos bem genéricos nesse aspecto, pois nem sempre vitórias são necessárias para a obtenção de algum título de campeonato. Em alguns casos, combinações de empates podem ser suficientes. Além disso, títulos não são os únicos objetivos dos torcedores. Classificações para campeonatos continentais (Liga dos Campeões, na Europa, e Libertadores da América, na América do Sul, são exemplos) também são importantes conquistas no futebol atual.

8 Dois comentários são importantes sobre as limitações do modelo. Embora os resultados positivos de um clube de futebol não dependam apenas da vontade dos seus dirigentes (jogadores e funcionários, por exemplo, também são partes essenciais), podemos assumi-los como representantes de todos aqueles que influenciam vitórias ou derrotas. Em outras palavras, existe uma relação de agente-principal entre a torcida e qualquer indivíduo que afete o resultado do clube dentro de campo. Além disso, é claro que não basta a vontade do dirigente para que o clube vença. Contudo, empiricamente vemos que torcedores reconhecem o seu esforço. Logo, a sua vontade de vencer basta para a satisfação dos torcedores.

9 É possível, também, imaginar um número qualquer de dirigentes, divididos em dois grupos, eficientes e ineficientes. Também, assumiremos que os dirigentes se distinguem apenas pela eficiência. Não consideraremos, por exemplo, corrupção. 
se o dirigente é eficiente (custo baixo $\underline{\theta}$ ) ou ineficiente (custo alto $\bar{\theta}$ ). Contudo, de conhecimento comum o custo fixo $\mathrm{F}$, que é o mesmo para ambos os tipos, e que o custo marginal $\theta \in \Theta=\{\theta, \bar{\theta}\}$. Adicionalmente, assumiremos que a estrutura de informação é exogenamente dada e que o agente é do tipo $\underline{\theta}$ com probabilidade p e do tipo $\bar{\theta}$ com probabilidade 1-p. Portanto, temos as funções custos

$$
\mathrm{C}(\mathrm{m}, \theta)=\underline{\theta} \mathrm{m}+\mathrm{F}
$$

com probabilidade $\mathrm{p}$ ou

$$
\mathrm{C}(\mathrm{m}, \bar{\theta})=\bar{\theta} \mathrm{m}+\mathrm{F}
$$

com probabilidade 1-p. Podemos aproveitar e introduzir um conceito que nos será muito útil mais à frente.

Definição 1. O spread da incerteza sobre o custo do dirigente é dada pela diferença entre seus tipos, tal que $\Delta \theta=\bar{\theta}-\underline{\theta}>0$.

É importante observar que as variáveis que serão consideradas no contrato entre as partes são m e g, onde esta última é a transferência monetária da torcida para os dirigentes, referente à compra de camisetas e outros artigos do clube, de ingressos e pagamento de mensalidades. Em especial, o aumento no número de sócios pode representar bem essa variável. Por hipótese, ambas variáveis são observáveis. Quanto à verificabilidade, por se tratar de um contrato informal, não existe uma terceira parte, externa, capaz de impor penalidades no caso de descumprimento do acordo. Ao contrário, vamos considerar que as próprias partes envolvidas sejam capazes de assumir essa tarefa. Aqui podemos considerar as manifestações citadas na introdução como exemplos. Seja $\Psi$ o conjunto de alocações possíveis, formalmente temos

$$
\Psi=\left\{(\mathrm{m}, \mathrm{g}): \mathrm{m} \in \mathbb{R}_{+}, \mathrm{g} \in \mathbb{R}\right\}
$$

Note também que estamos assumindo que o agente deriva utilidade dos gastos da torcida. Essa utilidade indiretamente pode estar ligada ao status que o dirigente obtém frente aos demais membros do clube, visando alcançar cargos em níveis mais elevados, ou mesmo, como é o caso em alguns clubes brasileiros atualmente, à remuneração direta ${ }^{10}$.

10 Parece ser uma tendência atual a contratação de dirigentes remunerados. Clubes como Grêmio e Vasco da Gama são pioneiros neste aspecto. 


\subsection{O contrato ótimo com informação completa}

Suponhamos, primeiramente, que não exista assimetria de informação entre a torcida e o dirigente. Então, o problema da torcida é livre de incerteza. Para uma delegação de tarefas com sucesso, a torcida deve oferecer a cada tipo de dirigente um nível de utilidade ao menos tão alto quanto ele obteria em uma ocupação alternativa. Essa é nossa restrição de participação. Se normalizarmos a utilidade de reserva (custo de oportunidade do trabalho alternativo) do dirigente para zero, as restrições tomam a seguinte forma

$$
\begin{aligned}
& \underline{\mathrm{g}}-\underline{\theta \mathrm{m}} \geq 0 \\
& \overline{\mathrm{g}}-\bar{\theta} \overline{\mathrm{m}} \geq 0
\end{aligned}
$$

onde fizemos $\mathrm{F}=\mathrm{O}$ a título de simplificação, pois como o custo fixo $\mathrm{F}$ é comum aos tipos de dirigentes, ele não afeta os resultados. É imediato que os níveis eficientes de produção de resultados positivos são obtidos por igualar o valor marginal da torcida ao custo marginal de cada dirigente ${ }^{11}$. Esse resultado servirá de parâmetro de comparação mais a frente e é mais bem definido abaixo.

Definição 2.As produções de resultados positivos com informação completa do dirigente eficiente $\mathrm{m}^{*}$ e ineficiente $\overrightarrow{\mathrm{m}}^{*}$ são first-best se atenderem, respectivamente, as seguintes condições

$$
\begin{aligned}
& \mathrm{V}^{\prime}\left(\underline{\mathrm{m}}^{*}\right)=\underline{\theta} \\
& \mathrm{V}^{\prime}\left(\overline{\mathrm{m}}^{*}\right)=\bar{\theta}
\end{aligned}
$$

$\mathrm{e}$

Em termos de bem-estar, os níveis de produção com informação completa $\underline{\mathrm{m}}^{\text {* }}$ e $\overline{\mathrm{m}}^{*}$ devem ser alcançados sempre que seus valores sociais, $\underline{W^{*}}=\mathrm{V}\left(\mathrm{m}^{*}\right)-\underline{\theta \mathrm{m}^{*}}-\mathrm{F}$ e $\overline{\mathrm{W}}^{*}=\mathrm{V}\left(\overline{\mathrm{m}}^{*}\right)-\bar{\theta} \overline{\mathrm{m}}^{*}-\mathrm{F}$, respectivamente, forem não negativos. A proposição 3 abaixo e o seu subsequente corolário apresentam um resultado menos exigente.

Proposição 3. Em um ambiente de informação simétrica, os dirigentes eficientes geram mais bem-estar para a sociedade do que os ineficientes, ou seja, $\underline{W^{*}}>\underline{W^{*}}$.

Prova. Demonstraremos que $\mathrm{V}\left(\mathrm{m}^{*}\right)-\underline{\theta \mathrm{m}^{*}}>\mathrm{V}\left(\overline{\mathrm{m}}^{*}\right)-\bar{\theta} \overline{\mathrm{m}}^{*}$, o que é equivalente a demonstrar que ${\underline{w^{*}}}^{*}{\underline{W^{*}}}^{*}$. Por definição, $\underline{\mathrm{m}}^{*}$ maximiza $\mathrm{V}(\mathrm{m})-\underline{\theta} \mathrm{m}$ , o que implica $\mathrm{V}\left(\underline{\mathrm{m}}^{\prime}\right)-\underline{\theta \mathrm{m}^{\prime}} \geq \mathrm{V}(\mathrm{m})-\underline{\theta} \mathrm{m}$. Ainda, sabendo que $\bar{\theta}>\underline{\theta}$, temos $\mathrm{V}\left(\mathrm{m}^{*}\right)-\underline{\theta \mathrm{m}^{*}} \geq \mathrm{V}\left(\overline{\mathrm{m}}^{*}\right)-\underline{\underline{\theta}} \overline{\mathrm{m}}^{*}>\mathrm{V}\left(\overline{\mathrm{m}}^{*}\right)-\bar{\theta} \overline{\mathrm{m}}$.

Corolário 4. Para que as produções ocorram basta que a produção do tipo 11 Ver apêndice matemático. 
menos eficiente tenha valor social, tal que

$$
\overline{\mathrm{W}}^{*}=\mathrm{V}\left(\overline{\mathrm{m}}^{*}\right)-\bar{\theta} \overline{\mathrm{m}}^{*} \geq \mathrm{F}
$$

Prova. Segue imediatamente da proposição 3.

Para implementar os níveis de produção de first-best, a torcida pode fazer a seguinte tipo de oferta, pegar-ou-largar, para o dirigente: se $\theta=\bar{\theta}$ (respectivamente $\underline{\theta})$, a torcida gasta $\overline{\mathrm{g}}^{*}\left(\underline{\mathrm{g}}^{*}\right)$ para o nível de produção $\overline{\mathrm{m}}^{*}\left(\underline{\mathrm{m}}^{*}\right)$ com $\overline{\mathrm{g}}^{*}=\bar{\theta} \overline{\mathrm{m}}^{*}\left(\mathrm{~g}^{*}=\underline{\theta \mathrm{m}^{*}}\right)$. Independente do seu tipo, o dirigente aceita a oferta e obtém bem-estar zero. Assim, os contratos ótimos sob informação completa são $\left(\mathrm{g}^{*}, \underline{\mathrm{m}}^{*}\right)$ se $\theta=\underline{\theta}$ e $\left(\overline{\mathrm{g}}^{*}, \overline{\mathrm{m}}^{*}\right)$ se $\theta=\bar{\theta}$. A próxima proposição resume os principais resultados do contrato ótimo com informação completa.

Proposição 5. Sob informação simétrica, o menu ótimo de contratos tem as seguintes características:

i. A quantidade de bons resultados produzidos pelo dirigente eficiente $\underline{\mathrm{m}}$ "é estritamente maior que a quantidade produzida pelo dirigente ineficiente $\overline{\mathrm{m}}^{*}$, ou seja, $\underline{\mathrm{m}}^{*}>\overline{\mathrm{m}}^{*}$.

ii. Ambos os tipos de dirigente obtém utilidade igual a zero e os gastos ótimos da torcida em produtos do clube são $\underline{\mathrm{g}}^{*}=\underline{\theta \mathrm{m}}^{*} \mathrm{e} \overrightarrow{\mathrm{g}}^{*}=\bar{\theta} \overline{\mathrm{m}}^{*}$ para o os tipos eficiente e ineficiente, respectivamente.

Prova. Para demonstrar o item $i$, note que $V^{\prime}\left(\underline{m}^{*}\right)<V^{\prime}\left(\bar{m}^{*}\right)$, já que $\bar{\theta}>\underline{\theta}$. Segue, então, que $\underline{m}^{*}>\bar{m}^{*}$, pois $V^{\prime \prime}<0$. Já o item ii é demonstrado por substituir $\mathrm{g}^{*} \mathrm{e}$ $\overline{\mathrm{g}}^{*}$ em (4) e (5).

É importante ressaltar que a delegação da obtenção de bons resultados por parte da torcida para os dirigentes no contexto de informação completa ocorre sem custo nenhum para os torcedores. O principal obtém a mesma utilidade que obteria caso ele mesmo efetuasse a tarefa, ou seja, em ambientes como esse, por conhecer o tipo do dirigente, se eficiente ou não, o torcedor pode implementar um contrato que alcance exatamente seus desejos.

\subsection{O Contrato ótimo com informação incompleta}

Agora, admitamos que o custo marginal $\theta$ é informação privada do agente, a torcida não pode saber a priori se o dirigente é eficiente ou ineficiente. Note que o fato de possuir uma informação a qual o principal desconhece pode criar um incentivo ao agente não dizer a verdade, se isto lhe trouxer benefícios. Este tipo de situação quando aplicada a um ambiente com informação privada pode ser contornada se impormos uma restrição a mais no problema: a restrição de compatibilidade de incentivos. Formalmente, no nosso problema 
as restrições de compatibilidade de incentivos são

$$
\underline{g}-\underline{\theta m} \geq \bar{g}-\underline{\theta m}
$$

$\mathrm{e}$

$$
\overline{\mathrm{g}}-\bar{\theta} \overline{\mathrm{m}} \geq \underline{\mathrm{g}}-\bar{\theta} \underline{\mathrm{m}}
$$

Ao incluir estas duas restrições adicionais, não damos mais incentivos a nenhum dos tipos a mentir. A primeira inequação, por exemplo, estabelece que o dirigente eficiente terá uma utilidade maior ao falar a verdade do que ao se passar por ineficiente, enquanto a segunda faz o mesmo para o tipo ineficiente. Além das restrições de compatibilidade de incentivos (9) e (10), para o contrato ser aceito ainda temos as duas já conhecidas restrições de participação (4) e (5).

Juntas, as desigualdades (4), (5), (9) e (10), caracterizam o conjunto de alocações possíveis compatível em incentivos ${ }^{12}$. Este conjunto incorpora as restrições adicionais que devem ser feitas para se obter o contrato ótimo entre a torcida e o dirigente sob informação assimétrica ${ }^{13}$. Antes de passarmos a derivação do contrato ótimo, introduziremos o importante conceito de rendas de informação.

Definição 6. Se a quantidade de utilidade obtida pelo dirigente for superior à sua utilidade de reserva, dizemos que ele recebe uma renda de informação ou renda informacional. Sob nossa hipótese de utilidade de reserva normalizada para zero, existe renda informacional sempre que $U>0$.

Como visto no contrato ótimo sob informação completa, a torcida mantinha os dois tipos de dirigente a um nível zero de utilidade. Tínhamos um ambiente com renda informacional zero, ou seja,

\footnotetext{
12 Estamos assumindo válida uma restrição que garante a existência dos contratos ótimos. Esta é conhecida como restrição de monotonicidade e quando temos apenas dois tipos tem o simples formato de $\underline{m} \geq \overline{\mathrm{m}}$, ou seja, a produção do tipo mais eficiente deve ser igual ou superior ao do ineficiente. De fato, se esta condição se mantiver então $\bar{\theta}(\overline{\mathrm{m}}-\underline{\mathrm{m}}) \leq \overline{\mathrm{g}}-\underline{\mathrm{g}} \leq \underline{\theta}(\overline{\mathrm{m}}-\underline{\mathrm{m}})$, garantindo a existência de $\mathrm{g}^{*}$.

13 Existem dois casos especiais de menu de contratos que merecem ser citados. O primeiro deles é conhecido como pooling de contratos, pois os contratos de cada tipo coincidem. As restrições de compatibilidade de incentivos são trivialmente satisfeitas por esses contratos, mas existe uma perda de flexibilidade em relação à alocação de recursos que deixam de ser dependentes do estado da natureza. Com pooling de contratos só a restrição de participação importa. O segundo acontece quando um dos contratos é o contrato nulo $(0,0)$ e o contrato não nulo $\left(\mathrm{g}^{\mathrm{s}}, \mathrm{m}^{\mathrm{s}}\right)$ é aceito somente pelo tipo eficiente. Assim, (4) e (5) reduzem-se para $\mathrm{g}^{\mathrm{s}}-\underline{\theta} \mathrm{m}^{\mathrm{s}} \geq 0$ . Já a restrição de compatibilidade de incentivos do tipo ineficiente torna-se $0 \geq \mathrm{g}^{\mathrm{s}}-\bar{\theta} \mathrm{m}^{\mathrm{s}}$. Se tal desigualdade é estrita, então somente o tipo eficiente aceita o contrato. O benefício de tal menu é a redução do número de restrições, em contrapartida, todavia, pode se ter o custo da excessiva filtragem entre os tipos.
} 


$$
\mathrm{U}^{*}=\underline{\mathrm{g}}^{*}-\underline{\theta \mathrm{m}^{*}}=0
$$

$\mathrm{e}$

$$
\overline{\mathrm{U}}^{*}=\overline{\mathrm{g}}^{*}-\overline{\theta \mathrm{m}}^{*}=0
$$

Entretanto, em um ambiente com informação assimétrica essas igualdades dificilmente se manterão. Tomemos um menu de contrato qualquer $\{(\mathrm{g}, \mathrm{m}),(\overline{\mathrm{g}}, \overline{\mathrm{m}})\}$, então consideremos o nível de utilidade obtido pelo dirigente eficiente ao imitar o dirigente ineficiente

$$
\overline{\mathrm{g}}-\underline{\theta} \overline{\mathrm{m}}=\overline{\mathrm{g}}-\bar{\theta} \overline{\mathrm{m}}+\Delta \theta \overline{\mathrm{m}}=\overline{\mathrm{U}}+\Delta \theta \overline{\mathrm{m}}
$$

Onde utilizamos o conceito de spread da incerteza sobre o custo do dirigente.

Perceba que mesmo com a nossa hipótese simplificadora de $\overline{\mathrm{U}}=0$, o dirigente eficiente aufere uma utilidade positiva por se passar por ineficiente, proveniente, notadamente, das rendas de informação $\Delta \theta \overline{\mathrm{m}}$. Sempre que a torcida quiser que o dirigente ineficiente produza bons resultados na quantidade $\underline{\mathrm{m}}>\mathrm{o}$, ele também deverá dar uma renda de informação positiva para o agente eficiente. A quantidade total de bons resultados produzida diminuirá, dado que $2 \overline{\mathrm{m}}^{\prime \prime} \leq \underline{\mathrm{m}}^{*}+\overline{\mathrm{m}}^{*}$. Essa renda de informação é gerada pela vantagem informacional do agente frente ao principal. O problema do principal, nesse contexto, é encontrar o melhor modo de alocar essa renda dado qualquer conjunto de alocações compatível em incentivos possível.

De acordo com o timing implícito do jogo, a torcida oferece um menu de contratos antes de saber de que tipo é o dirigente, se eficiente ou ineficiente. Portanto, ela irá computar os benefícios de um menu qualquer em termos de expectativa. O problema da torcida então é

$$
\begin{aligned}
& \{(g, m),(\overline{g, m})\}^{p[V(\underline{m})-\underline{g}]+(1-p)[V(\bar{m})-\bar{g}]} \\
& \text { sujeito a (4), (5), (9) e (10). }
\end{aligned}
$$

Se usarmos as definições de rendas informacionais $\underline{\mathrm{U}}=\underline{\mathrm{g}}-\underline{\theta \mathrm{m}}$ e $\overline{\mathrm{U}}=\overline{\mathrm{g}}-\overline{\theta \mathrm{m}}$, podemos substituir na função objetivo da torcida os gastos da torcida g como função das rendas de informação e da quantidade de bons resultados, tal que no novo problema de otimização as variáveis de escolha são $\{(\underline{\mathrm{U}}, \underline{\mathrm{m}}),(\overline{\mathrm{U}}, \overline{\mathrm{m}})\}$. Ao focarmos nas rendas informacionais damos ênfase ao impacto distributivo da informação assimétrica. A nova função objetivo torna-se 


$$
\underbrace{\mathrm{p}[\mathrm{V}(\underline{\mathrm{m}})-\underline{\theta \mathrm{m}}]+(\mathbf{1}-\mathrm{p})[\mathrm{V}(\overline{\mathrm{m}})-\bar{\theta} \overline{\mathrm{m}}]}_{\text {Eficiência Alocativa Esperada }}-\underbrace{[\mathrm{p} \underline{\mathrm{U}}+(\mathbf{1}-\mathrm{p}) \overline{\mathrm{U}}]}_{\text {Renda Informacional Esperada }}
$$

Esta nova expressão claramente mostra que a torcida deseja maximizar o valor social esperado do seu bem-estar menos a renda informacional esperada do dirigente. A torcida aceitaria uma distorção alocativa em troca de um decréscimo na renda informacional do dirigente. Se escrevermos as restrições de compatibilidade de incentivos (9) e (10) em função das rendas de informação teremos

$$
\underline{\mathrm{U}} \geq \overline{\mathrm{U}}+\Delta \theta \overline{\mathrm{m}}
$$

e

$$
\overline{\mathrm{U}} \geq \underline{\mathrm{U}}-\Delta \theta \underline{\mathrm{m}}
$$

Já as restrições de participação tornam-se

$$
\underline{\mathrm{U}} \geq \mathbf{O}
$$

e

$$
\overline{\mathrm{U}} \geq 0
$$

Dessa forma, o problema da torcida passa a ser

$$
\{(\mathrm{U}, m),(\overline{\mathrm{U}, m})\}^{p[V(\underline{m})-\underline{\theta m}]+(1-p)[V(\bar{m})-\overline{\theta m}]-[p \underline{U}+(1-p) \bar{U}]}
$$

sujeito a (16) e a (19). 
Para a resolução do problema da torcida adotaremos uma abordagem mais intuitiva. A derivação tradicional, através do método de Kuhn-Tucker, pode ser encontrada no apêndice matemático.

Tentaremos, primeiramente, restringir o número de restrições do problema. Se assumirmos que não estamos em um dos casos especiais citados na nota 13, com $\overline{\mathrm{m}}>0$, então a restrição (18) sempre será satisfeita. De fato, (16) e (19) implicam diretamente (18), pois $\underline{\underline{U}} \geq \overline{\mathrm{U}}+\Delta \theta \overline{\mathrm{m}} \geq 0$. Dessa forma, intuitivamente, se um menu de contratos é capaz de fazer o dirigente ineficiente alcançar sua utilidade de reserva, então também o fará para o dirigente que produz a um custo mais baixo. Adicionalmente, (17) é irrelevante, haja vista que, ao contrário do eficiente, não há vantagem nenhuma para o ineficiente em mentir seu tipo.

Perceba que através de argumentos econômicos chegamos a uma redução das restrições do problema, restando apenas (16) e (19).

Proposição 7. As restrições (16) e (19) são satisfeitas com igualdade no ponto de ótimo, tal que

$$
\underline{\mathrm{U}}=\Delta \theta \overline{\mathrm{m}}
$$

$\mathrm{e}$

$$
\overline{\mathrm{U}}=\mathbf{O}
$$

Prova. A prova se dá por contradição. Assuma que a segunda igualdade não se mantenha, de forma que $\bar{U}=\varepsilon>0$. Então, de (16) segue que a torcida poder diminuir $\overline{\mathrm{U}}$ em $\varepsilon$ e consequentemente também $\underline{\mathrm{U}}$ em $\varepsilon$, ganhando $\varepsilon$. Isto caracteriza que não se está no ótimo.

A proposição anterior nos permite substituir (22) e (23) em (21) e, assim, obter um problema de otimização irrestrito com apenas a quantidade de resultados positivos como variável de escolha:

$$
\{(\underline{\mathrm{m}}, \overline{\mathrm{m}})\}^{\mathrm{p}[\mathrm{V}(\underline{\mathrm{m}})-\underline{\theta \mathrm{m}}]+(1-\mathrm{p})[\mathrm{V}(\overline{\mathrm{m}})-\bar{\theta} \overline{\mathrm{m}}]-\mathrm{p} \Delta \theta \overline{\mathrm{m}}}
$$

Compare (24) com o problema com informação completa. Observe que a única diferença é a presença do termo de renda informacional esperada do dirigente eficiente subtraindo o problema de otimização. Veja também que o montante de renda transferida será proporcional a produção de bons resultados pelo 
dirigente ineficiente.

As condições de primeira ordem de (24) são

$$
\mathrm{V}^{\prime}\left(\underline{\mathrm{m}}^{\mathrm{SB}}\right)=\underline{\theta} \quad \text { ou } \quad \underline{\mathrm{m}}^{\mathrm{SB}}=\underline{\mathrm{m}}^{*}
$$

e

$$
(1-\mathrm{p})\left[\mathrm{V}^{\prime}\left(\overline{\mathrm{m}}^{\mathrm{SB}}\right)-\bar{\theta}\right]=\mathrm{p} \Delta \theta
$$

É imediato que a quantidade ótima de produção de bons resultados pelo dirigente eficiente é a mesma tanto sob informação simétrica quanto assimétrica (o sobrescrito SB se refere a second-best) no contrato ótimo. Esse resultado é garantido pela transferência da renda informacional. Todavia, a produção de resultados positivos pelo dirigente ineficiente será inferior ao first-best. Podemos resumir as principais características do contrato ótimo sob informação assimétrica na proposição a seguir ${ }^{14}$.

Proposição 8. Sob informação assimétrica, o menu ótimo de contratos tem as seguintes características:

i. Não há distorção na quantidade de bons resultados produzidos pelo dirigente eficiente em relação ao first-best, $\underline{\mathrm{m}}^{\mathrm{sB}}=\underline{\mathrm{m}}^{*}$. Porém há uma distorção negativa para o dirigente ineficiente, $\overline{\mathrm{m}}^{\mathrm{SB}}<\overline{\mathrm{m}}^{*}$ respeitando

$$
\mathrm{V}^{\prime}\left(\overline{\mathrm{m}}^{\mathrm{SB}}\right)=\bar{\theta}+\frac{\mathrm{p}}{1-\mathrm{p}} \Delta \theta
$$

ii. Somente o tipo eficiente obtém uma positiva renda informacional dada por

$$
\underline{\mathrm{U}}^{\mathrm{SB}}=\Delta \theta \overline{\mathrm{m}}^{\mathrm{SB}}
$$

iii. Os gastos da torcida no resultado de second-best são respectivamente dados por $\underline{\mathrm{g}}^{\mathrm{SB}}=\underline{\theta \mathrm{m}}^{*}+\Delta \theta \overline{\mathrm{m}}^{\mathrm{SB}}$ e $\overline{\mathrm{g}}^{\mathrm{SB}}=\bar{\theta} \overline{\mathrm{m}}^{*}$.

14 Já que resolvemos o problema de modo intuitivo, restringindo o número de restrições, devemos verificar se a restrição de compatibilidade de incentivos do ineficiente, excluída, é atendida no ótimo. Em outras palavras, desejamos saber se $0 \geq \Delta \theta\left(\overline{\mathrm{m}}^{\mathrm{SB}}-\underline{\underline{m}}^{\mathrm{SB}}\right)$ se mantém. De fato, dado que $\underline{m}^{\mathrm{SB}}=\underline{m}^{*}>\overline{\mathrm{m}}^{*}>\overline{\mathrm{m}}^{\mathrm{SB}}$, a restrição é atendida. 
Prova. No item $i, \underline{\mathrm{m}}^{\mathrm{SB}}=\underline{\mathrm{m}}^{*}$ segue de (25). Para demonstrar que $\overline{\mathrm{m}}^{\mathrm{SB}}<\overline{\mathrm{m}}^{*}$ basta notarmos que $\frac{\mathrm{p}}{1-\mathrm{p}} \Delta \theta>0$ e que $\mathrm{V}^{\prime \prime}<0$. O resultado $i i$ é imediato da proposição 7. Já iii é logicamente equivalente a ii, basta usarmos a definição de renda informacional.

\section{3•3. Alguns resultados teóricos}

A questão a ser respondida agora é a seguinte: em qual dos dois modelos apresentados encaixasse o futebol brasileiro? Se a resposta é o primeiro caso, onde temos informação simétrica, teríamos nos nossos clubes um resultado de first-best. Então, o contrato ótimo geraria a quantidade de resultados positivos exatamente igual àquela desejada pelos torcedores e não haveria incompatibilidade entre os seus interesses e os da direção. Também, sob estas circunstâncias, ambos os tipos de dirigente obteriam bem-estar igual ao seu nível de reserva.

Parece claro que este não é o ambiente que vigora no país. Com relação à quantidade ótima de resultados positivos, por exemplo, se estes fossem de first-best, então não haveria motivo para manifestações dos torcedores. Todavia, conforme as notícias apresentadas na introdução, este tipo de ação por parte da torcida é uma prática recorrente no Brasil. Perceba que ao compararmos a quantidade de bons resultados produzida em informação completa e incompleta, com e sem restrição de compatibilidade de incentivos, $\underline{\underline{m}}^{*}+\overline{\mathrm{m}}>\underline{\underline{m}}+\overline{\mathrm{m}}^{\mathrm{sB}}>2 \overline{\mathrm{m}}^{*}$, podemos ver que não vão conseguir implementar o contrato ótimo, a torcida tem uma perda de bem-estar. Quanto a segunda consequência do first-best, a de que os dirigentes obtêm um nível de utilidade iguala sua quantidade de reserva (na nossa simplificação $\overline{\mathrm{U}}=\underline{\mathrm{U}}=0$ ), esta parece também não severificar, já que alguns fatos parecem apontar na direção contrária, como as acirradas disputaseleitorais nos clubes brasileiros nos últimos anos. Se os dirigentes recebessem apenas a utilidade de reserva, eles deveriam ser indiferentes entre trabalhar no clube ou em alguma ocupação alternativa.

Agora que concluímos que o ambiente no qual a torcida deve tomar decisões é de informação incompleta, vamos permitir que o dirigente tente revelar seu tipo. Pode-se pensar que é benéfico para os dirigentes que haja uma sinalização através dos gastos com campanha eleitoral, por exemplo, pois o resultado para a sociedade como um todo é melhor sob informação completa. Entretanto, como mostra a proposição a seguir, isto não é verdade, individualmente, para nenhum dos tipos.

Proposição 9. Seja $\mathrm{T}$ o gasto do dirigente com campanha eleitoral (ou qualquer outro gasto com sinalização). Então, $\mathrm{T}=\mathrm{O}$ para ambos os tipos de dirigente. Em outras palavras, sob as condições dadas no problema com informação assimétrica, não é racional sinalizar. 
Prova. Suponha, por absurdo, que o dirigente eficiente sinalize. Para o ineficiente o argumento é trivial, já que recebe a utilidade de reserva em ambos os casos. Então, o dirigente eficiente poderia escolher $0<\mathrm{T}<\Delta \theta \overline{\mathrm{m}}^{-\mathrm{St}}$. Contudo, dessa forma, com a torcida podendo diferenciar os tipos, o resultado seria o de first-best, que é pior para o dirigente eficiente, apesar de socialmente desejável.

Esta proposição nos mostra que não existe uma maneira de os torcedores chegarem ao first-best (estamos desconsiderando screening aqui, pois é difícil imaginar em algo implementável por parte da torcida). Portanto, a torcida possui somente duas opções sobre informação assimétrica: a implementação do contrato de second-best, dados pela proposição 7, ou o resultado no qual o tipo eficiente imita o ineficiente, citado anteriormente. Como sabemos, a diferença entre eles está na ausência da compatibilidade de incentivos no segundo.

É razoável supor, portanto, que a torcida desejará implementar o menu de contratos de second-best. Isto é verdadeiro pois, por hipótese, os torcedores e dirigentes conseguem observar em que ambientes estão, se sob informação simétrica ou assimétrica. Contudo, pode ocorrer de não ser possível impor as restrições de compatibilidade de incentivos ou, principalmente, verificar se ela está sendo atendida. Isto pode ocorrer talvez por não existir um órgão externo capaz de impor punições no caso de descumprimento.

Dessa forma, conseguimos explicar de uma maneira teórica uma razão para manifestações tão violentas por parte de torcedores dos clubes brasileiros: a divergência entre seus desejos, na maioria das vezes vitórias e resultados positivos, e os da direção, os quais podem ir desde manutenção do orçamento ao aumento do número de sócios do clube. De fato, sempre que o contrato de second-best não puder ser implementado, pelas razões discutidas anteriormente, haverá reação por parte dos torcedores. Esse resultado pode também lançar luz na questão de que por que alguns times sofrem mais com invasões de campo e outros tipos de manifestações agressivas do que outros. Pelo o modelo desenvolvido aqui, a causa é que nos primeiros o contrato de second-best não consegue ser implementado, enquanto que nos últimos consegue.

\section{Conclusão}

Em vista da escassez da literatura internacional sobre o tema, o presente trabalho objetivou contribuir para o desenvolvimento da ainda nascente Economia do Futebol e, principalmente, desenvolver um simples modelo teórico que focasse a relação de agência existente entre torcida e dirigentes de futebol no Brasil. Em um ambiente de informação incompleta, a torcida, representando o papel do principal, delega a tarefa da produção de vitórias e bons resultados para o dirigente, o agente, sem saber seu tipo, se eficiente ou ineficiente. Seu desafio é implementar o contrato de second-best, fazendo 
com que as restrições de compatibilidade de incentivos sejam atendidas, já que a ausência de uma terceira parte, externa, tal como uma corte de justiça, capaz de garantir os contratos, torna esta tarefa complicada.

As principais conclusões teóricas que o modelo fornece encontram embasamento nas evidências empíricas. Primeiramente, podemos entender as agressões a dirigentes, jogadores e técnicos de futebol e as invasões de campo protagonizadas por torcedores. Pelo nosso modelo, sempre que a torcida não implementa o resultado de second-best, seja por não conseguir garantir a execução da restrição da compatibilidade de incentivos seja por algum outro motivo contratual, há uma divergência de objetivos entre as partes e, como resultado, uma manifestação violenta dos torcedores. Adicionalmente, como o modelo indica que isto não será verdade em todos os casos, somente quando a relação contratual não for verificada, temos argumentos para justificar a disparidade das reações de torcedores em diferentes equipes. Alguns, como os times de massa citados na introdução, sofrem com manifestações frequentemente, enquanto outros clubes raramente. Segundo o modelo, isto acontece pois, enquanto nos últimos o contrato de second-best vigora, no primeiro ele não consegue ser implementado.

Algumas interessantes extensões nesta área de Economia do Futebol podem ser feitas. Uma possibilidade imediata é a inclusão de seleção adversa no modelo, nos casos onde a escolha da direção do clube pode ser feita através do voto dos sócios. Por ser informação privada do dirigente, o torcedor não observa o seu tipo, apenas a média, e dessa forma pode-se recair no problema dos lemons. Outro possível incremento diz respeito à influência direta $\mathrm{e}$ parcial do principal no resultado. Intuitivamente, quanto maior a torcida no estádio assistindo o jogo maior a probabilidade de vitória do time da casa. Aqui pode existir uma relação estratégica entre agente e principal, com o segundo podendo diminuir seu esforço, por exemplo, no caso de influência positiva direta do agente sobre o resultado. Um arcabouço de teoria dos jogos parece adequado ao problema.

A abordagem através de desenho de mecanismos também pode ser muito útil nas circunstâncias do esporte, particularmente no futebol. A complexidade do tema e suas peculiaridades informacionais merecem uma estrutura formal mais rigorosa. Uma extensão em especial, contudo, merece ser considerada. Esta é a modelagem da própria não verificabilidade do contrato. Em outras palavras, um modelo ideal precisa incorporar como uma de suas partes explícitas a ausência de uma instituição externa capaz de fazer cumprir os acordos e verificar todos os parâmetros do problema. Através dessa inclusão seria possível tirar conclusões sobre suas influências diretas no contrato ótimo e ainda relaxar algumas hipóteses mais rígidas do modelo. 


\section{Referências}

El-Hodiri, M. \&Quirk, J. (1971). An Economic Model of a Professional Sports League. Journal of Political Economy, 79 (6): 1302-1319.

Fort, R. (2000). European and North American Sports Differences (?). Scottish Journal of Political Economy, 47 (4): 431-455.

Giovannetti, B., Rocha, B., Sanches, F. \& Silva, J. C. D. (2006). Medindo a Fidelidade das Torcidas Brasileiras: Uma Análise Econômica do Futebol. Revista Brasileira de Economia, 60(4): 389-406.

Laffont, J. \& Martimort, D. (2002). The Theory of Incentives: The Principal-Agent Model. Princeton University Press.

Miceli, T. J. (2004). A principal-Agent Model of Contracting in Major League Baseball. Journal of Sports Economics, 5 (2): 213-220.

Neale, W. (1964). The Peculiar Economics of Professional Sports. Quarterly Journal of Economics, 78 (1): 1-14.

Rottenberg, S. (1956). The Baseball Players' Labor Market. Journal of Political Economy, 64 (3): 242-258.

Sloane, P. (1971). The Economics of Profesional Soccer: The Soccer Team as a Utility Maximizer. Scottish Journal of Political Economy, 18 (2): 121-146.

Sloane, P. (2006). Rottenberg and the Economics of Sport after 50 Years: An Evaluation. IZA Discussion Paper n. 2175.

Vrooman, J. (2007). Special Issue on Economics of Sport: Editor's Introduction. Scottish Journal of Political Economy, 54 (3): 314-317.

\section{Apêndice matemático}

Aqui apresentamos a resolução do problema do principal pelo método tradicional do lagrangeano. Como o problema inclui restrições de desigualdade, utilizaremos o método de Kuhn-Tucker.

\section{Informação completa}

Em um ambiente de informação simétrica temos que o problema do principal é:

$$
\begin{aligned}
& \{(m, g),(\bar{m}, \bar{g})\}^{V(\underline{m})-\underline{g}+V(m)-g} \\
& \text { sujeito a (4) e (5), }
\end{aligned}
$$

onde adicionamos as condições de não negatividade $\underline{m}, \underline{g}, \bar{m}, \bar{g} \geq 0$. 
O lagrangeano, então, toma a seguinte forma

$$
L\left(\underline{m}, \underline{g}, \bar{m}, \bar{g}, \lambda_{1}, \lambda_{2}\right)=V(\underline{m})-\underline{g}+V(\bar{m})-\bar{g}+\lambda_{1}(\underline{g}-\underline{\theta m})+\lambda_{2}(\bar{g}-\overline{\theta m})
$$

e suas condições de primeira ordem são

$$
\begin{gathered}
\frac{\partial L}{\partial \underline{m}}=V^{\prime}(\underline{m})-\lambda_{1} \underline{\theta} \leq 0, \underline{m} \geq, \frac{\partial L}{\partial \underline{m}} \underline{m}=0 \\
\frac{\partial L}{\partial \bar{m}}=V^{\prime}(\bar{m})-\lambda_{2} \bar{\theta} \leq 0, \bar{m} \geq, \frac{\partial L}{\partial \bar{m}} \bar{m}=0 \\
\frac{\partial L}{\partial g}=-1+\lambda_{1} \leq 0, \underline{g} \geq 0, \frac{\partial L}{\partial g}=0 \\
\frac{\partial L}{\partial \bar{g}}=-1+\lambda_{2} \leq 0, \bar{g} \geq 0, \frac{\partial L}{\partial \bar{g}}=0 \\
\frac{\partial L}{\partial \lambda_{1}}=g-\underline{\theta m} \geq 0, \lambda_{1} \geq 0, \frac{\partial L}{\partial \lambda_{1}} \lambda_{1}=0 \\
\frac{\partial L}{\frac{\partial \lambda}{\lambda_{2}}}=\bar{g}-\overline{\theta m} \geq 0, \lambda_{2} \geq 0, \frac{\partial L}{\partial \lambda_{2}} \lambda_{2}=0
\end{gathered}
$$

Primeiramente vamos considerar o caso onde todas as restrições são atendidas com igualdade e as condições de não-negatividade com desigualdade estrita. Pela condição de complementaridade do teorema de Kuhn-Tucker, isto implica que $\underline{m}, g, \bar{m}, \bar{g}, \lambda_{1}, \lambda_{2}>0$. Com isto, é imediato de (33) e (34) que $\lambda_{1}=\lambda_{2}=1$ e ao substituirmos em (31) e (32) temos

$$
\begin{aligned}
& V^{\prime}(\underline{m})=\underline{\theta} \\
& V^{\prime}(\bar{m})=\bar{\theta} .
\end{aligned}
$$

Ao aplicarmos o teorema da função inversa obtemos uma forma explícita para a quantidade ótima de resultados positivos abaixo (a existência da função inversa é garantida pelas hipóteses feitas sobre 


$$
\begin{aligned}
& \underline{m}{ }^{*}=V^{\prime-1}(\underline{\theta}) \\
& \bar{m}^{*}=V^{\prime-1}(\bar{\theta})
\end{aligned}
$$

A quantidade ótima de gasto da torcida é obtida diretamente de (35) e (36).

$$
\begin{aligned}
& \underline{g}^{*}=\underline{\theta m}{ }^{*} \\
& \bar{g}^{*}=\overline{\theta m}
\end{aligned}
$$

Informação incompleta

Já com informação assimétrica temos:

$$
\begin{aligned}
& \{(m, g),(\bar{m}, \bar{g})\}^{p[V(\underline{m})-\underline{g}]+(1-p)[V(\bar{m})-\bar{g}]} \\
& \text { sujeito a (4), (5), (9) e (10), }
\end{aligned}
$$

além das restrições de não-negatividade.

O lagrangeano tem a seguinte expressão:

$$
\begin{aligned}
& L\left(\underline{m}, \underline{g}, \bar{m}, \bar{g}, \mu_{1}, \mu_{2}, \mu_{3} \mu_{4}\right)=p[V(\underline{m})-\underline{g}]+(1-p)[V(\bar{m})-\bar{g}]+ \\
& \mu_{1}(\underline{g}-\underline{\theta m})+\mu_{2}(\bar{g}-\bar{\theta} \bar{m})+\mu_{3}(\underline{g}-\underline{\theta m}-\bar{g}+\underline{\theta} \bar{m})+\mu_{4}(\bar{g}-\bar{\theta} \bar{m}-\underline{g}+\underline{\theta m}) .
\end{aligned}
$$

As condições de primeira ordem são:

$$
\begin{gathered}
\frac{\partial L}{\partial \underline{m}}=p V^{\prime}(\underline{m})-\mu_{1} \underline{\theta}-\mu_{3} \underline{\theta}+\mu_{4} \bar{\theta} \leq \mathbf{0}, \underline{m} \geq \mathbf{0}, \frac{\partial L}{\partial \underline{m}} \underline{m}=\mathbf{O} \\
\frac{\partial L}{\partial \bar{m}}=(1-p) V^{\prime}(\bar{m})-\mu_{2} \bar{\theta}+\mu_{3} \underline{\theta}-\mu_{4} \bar{\theta} \leq 0, \bar{m} \geq 0, \frac{\partial L}{\partial \bar{m}} \bar{m}=0
\end{gathered}
$$




$$
\begin{gathered}
\frac{\partial L}{\partial \underline{g}}=-p+\mu_{1}+\mu_{3}-\mu_{4} \leq \mathrm{O}, \underline{g} \geq \mathrm{O}, \frac{\partial L}{\partial \underline{g}} \underline{g}=\mathrm{O} \\
\frac{\partial L}{\partial \bar{g}}=-(1-p)+\mu_{2}+\mu_{3}-\mu_{4} \leq \mathrm{O}, \bar{g} \geq \mathrm{O}, \frac{\partial L \overline{\bar{g}}}{\partial \boldsymbol{g}}=\mathrm{O} \\
\frac{\partial L}{\partial \mu_{1}}=\underline{g}-\underline{\theta m} \geq \mathrm{O}, \mu_{1} \geq \mathrm{O}, \frac{\partial L}{\partial \mu_{1}} \mu_{1}=\mathrm{O} \\
\frac{\partial L}{\partial \mu_{2}}=\bar{g}-\bar{\theta} \bar{m} \geq \mathrm{O}, \mu_{2} \geq \mathrm{O}, \frac{\partial L}{\partial \mu_{2}} \mu_{2}=\mathrm{O} \\
\frac{\partial L}{\partial \mu_{3}}=\underline{g}-\underline{\theta m}-\bar{g}+\underline{\theta m} \geq \mathrm{O}, \mu_{3} \geq \mathrm{O}, \frac{\partial L}{\partial \mu_{3}} \mu_{3}=\mathrm{O} \\
\frac{\partial L}{\partial \mu_{4}}=\bar{g}-\bar{\theta} \bar{m}-\underline{g}+\underline{\theta m} \geq \mathrm{O}, \mu_{4} \geq \mathrm{O}, \frac{\partial L}{\partial \mu_{4}} \mu_{4}=\mathrm{O}
\end{gathered}
$$

Aqui se a primeira tentativa for com todas as restrições sendo atendidas com igualdade, ou seja, $\underline{m}, \underline{g}, m, g, \mu_{1}, \mu_{2}, \mu_{3}, \mu_{4}>0$ chegaremos ao seguinte sistema a ser resolvido:

$$
\begin{array}{r}
p\left[V^{\prime}(\underline{m})-\underline{\theta}\right]+\mu_{4} \Delta \theta=\mathbf{O} \\
(1-p)\left[V^{\prime}(\bar{m})-\bar{\theta}\right]-\mu_{3} \Delta \theta=\mathbf{O}
\end{array}
$$

É fácil ver que é impossível resolvê-lo, além disso, as restrições formam um sistema sobreidentificado.

Vamos agora considerar então que duas das restrições não sejam atendidas com igualdade, de tal forma que $\mu_{1}=\mu_{4}=0$. Essas condições implicam $\mu_{3}=p$ e $\mu_{2}=1$. Ao substituirmos em (45) e (46), chegamos à quantidade ótima de second-best:

$$
V^{\prime}\left(\underline{m}^{S B}\right)=\underline{\theta}
$$


GRIEBELER, M. de C.; BALDUSCO, D. Economia do Futebol:Uma Abordagem Através do Modelo Agente-Principal

$$
V^{\prime}\left(\bar{m}^{S B}\right)=\bar{\theta}+\frac{p}{1-p} \Delta \theta
$$

Ao aplicarmos novamente o teorema da função inversa chega-se a

$$
\begin{gathered}
\underline{m}^{S B}=\underline{m}{ }^{*}=V^{\prime-1}(\underline{\theta}) \\
\bar{m}^{S B}=V^{\prime-1}\left(\bar{\theta}+\frac{p}{1-p} \Delta \theta\right)
\end{gathered}
$$

Já os valores das quantidades ótimas são obtidos das restrições atendidas por igualdade:

$$
\begin{gathered}
\underline{g}^{S B}=\underline{\theta m}+\bar{m} \Delta \theta \\
\bar{g}{ }^{S B}=\bar{\theta} \bar{m}
\end{gathered}
$$

Para ver que as restrições com desigualdade são atendidas no ótimo, veja a seção 3.2. 